\title{
The whole transcriptome and proteome changes in the early stage of myocardial infarction
}

\author{
Yanfei Li ${ }^{1}$, Cuiping Wang ${ }^{1}$, Tingting $\mathrm{Li}^{1}$, Linlin Ma', Fangzhou Fan', Yueling Jin ${ }^{1}$ and Junwei Shen ${ }^{2}$
}

\begin{abstract}
As the most severe manifestation of coronary artery disease, myocardial infarction (MI) is a complex and multifactorial pathophysiologic process. However, the pathogenesis that underlies MI remains unclear. Here, we generated a MI mouse model by ligation of the proximal left anterior descending coronary artery. The transcriptome and proteome, at different time points after Ml, were detected and analysed. Immune-related pathways, cell cycle-related pathways, and extracellular matrix remodelling-related pathways were significantly increased after MI. Not only innate immune cells but also adaptive immune cells participated in the early stage of Ml. Proteins that functioned in blood agglutination, fibrinolysis, secretion, and immunity were significantly changed after MI. Nppa, Serpina3n, and Anxa1, three secreted proteins that can easily be detected in blood, were significantly changed after MI. Our discoveries not only reveal the molecular and cellular changes in Ml but also identify potential candidate biomarkers of $\mathrm{Ml}$ for clinical diagnosis or treatment.
\end{abstract}

\section{Introduction}

Myocardial infarction (MI), the most severe manifestation of coronary artery disease, causes more than 6.4 million deaths in the US, Europe, and Northern Asia and more than a third of the deaths in developed nations each year ${ }^{1,2}$. Additionally, its medical burden is tremendous; in 2010, more than 1.1 million US hospitalisations were a result of MI, with estimated direct costs of at least US $\$ 450$ billion, but there were more than 2.4 million patients with MI in $2016^{3,4}$. Exploration of integrated cellular and molecular characteristics of MI may help to deepen the understanding of myocardial dysfunction during MI, leading to the establishment of personalised treatment and prevention strategies and improvements in patients' clinical outcomes ${ }^{4}$.

\footnotetext{
Correspondence: Yueling Jin (Jinyl@sumhs.edu.cn) or Junwei Shen (xingkezh5@163.com)

1Shanghai University of Medicine \& Health Sciences, Shanghai 201318, China ${ }^{2}$ Tongji University affiliated Eastern Hospital, Shanghai 200092, China Edited by A. Ruffini
}

MI is a complex process with different components, including cell hypoxia, apoptosis, migration, fibrosis, and immune cell infiltration. Zhu et al. suggested vascular endothelial cell migration and apoptosis that was induced by iNOS play a crucial role in $\mathrm{MI}^{5}$. Ma et al. have demonstrated a dramatic increase in autophagy during the reperfusion phase of cardiac ischaemia ${ }^{6}$. Prabhu et al. and Wang et al. revealed the participation of the inflammatory response in the early stage of $\mathrm{MI}^{7,8}$. However, the roles of immune cells in this process have not been identified clearly.

Protein detection plays a major part in medical testing. For example, cardiac troponin is an important diagnostic indicator in MI. Similarly, ANP and BNP are diagnostic markers for heart failure. The changes in protein concentrations during MI progression not only have good clinical research value but also have strong application prospects for clinical diagnosis and treatment. Mass spectrometry (MS) is a key technology of proteomics that is used to identify and quantify proteins and peptides. For example, top-down proteomics reveals that $\mathrm{ENH} 2$, which 
belongs to the PDZ-LIM protein family, is highly expressed in the early stage of MI and contributes to cardiac dysfunction ${ }^{9}$. Proteins implicated in vascular endothelial growth factor (VEGF) signalling and extracellular matrix are significantly up-regulated in the periinfarct border zone after $\mathrm{MI}^{10}$. One recent advance in MS-based targeted proteomics is data-independent acquisition mass spectrometry (DIA-MS) ${ }^{11}$. By characterising different molecules produced from different samples, this method combined with improved bioinformatics analysis is more sensitive in detecting peptides or proteins that are usually missed in traditional MS experiments. The present study is designed to investigate the cellular and molecular changes in different MI stages in a mouse model through the combination of transcriptome and proteome analyses.

\section{Results}

\section{Functional evaluation of MI mouse model}

To investigate the molecular changes of $\mathrm{MI}$ in vivo, C57 mice were treated with MI or sham operation. Then, the infarct left ventricle tissue and control tissue were acquired for RNA-seq and DIA-MS (Fig. 1a). The infarct regions were mainly in the ventricular apical region (Fig. 1b). Myocardial fibrosis is one of the main events after MI, so we evaluated the model by detecting the degree of myocardial fibrosis ${ }^{8,12}$. Masson staining displayed the fibres in the myocardium as blue, and the staining depth reflected the degree of fibrosis. Compared with the control myocardium, fibrotic aggregations were observed in some tissues after $24 \mathrm{~h}$ of MI treatment. Additionally, fibrosis was obvious in part of the cardiac tissue in the infarct area after $72 \mathrm{~h}$ of MI treatment. These results indicate that this mouse model effectively mimicked the MI process (Fig. 1c).

\section{Gene expression profiling analysis}

A total of 13,390 genes in the RNA-seq data were detected and analysed. The principal component analysis (PCA) of the RNA-seq data showed that the three groups of samples distinctively clustered into three different categories, indicating the gene expression pattern between these three groups was different (Fig. 2a). Then, we compared the differentially expressed genes (DEGs) and made a volcano plot. Compared with the control group, the gene expression displayed large differences in the $24 \mathrm{~h}$ group and the $72 \mathrm{~h}$ group (Fig. 2b). The Venn plot showed that the majority (80\%) of DEGs between control and $24 \mathrm{~h}$ groups were the same as those between control and $72 \mathrm{~h}$ groups (Fig. 2c). Finally, the heatmap data showed that the top 200 DEGs could significantly distinguish these three groups of tissues (Fig. 2d).
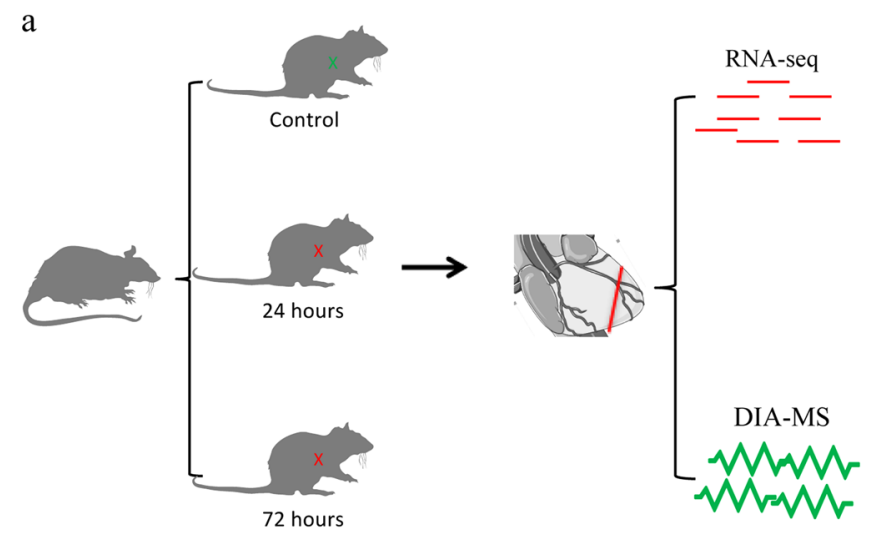

b

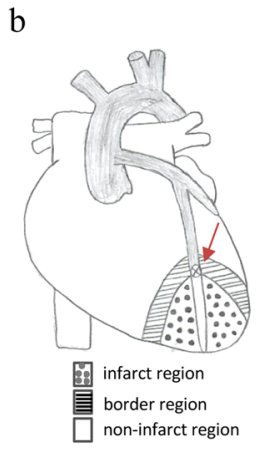

c

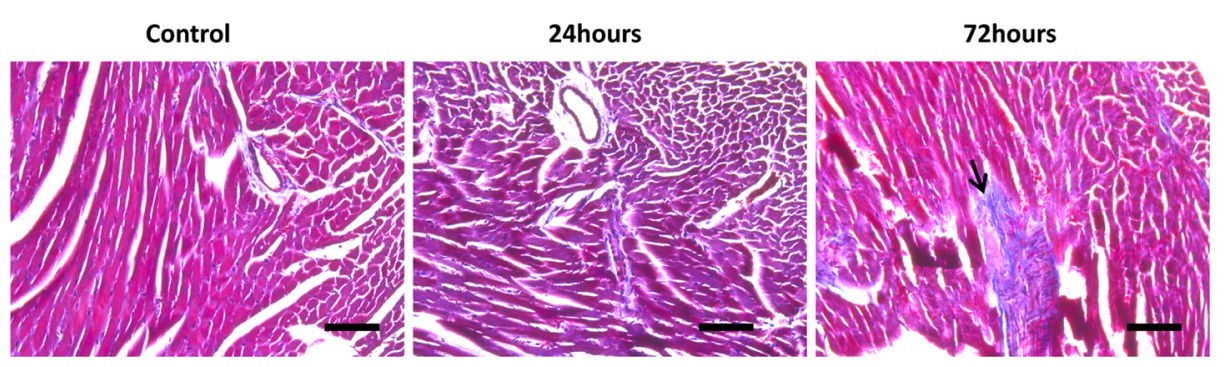

Fig. 1 Functional evaluation of myocardial infarction mouse model. a Schematic diagram of this study. $\mathbf{b}$ Schematic diagram of the mouse Ml model and infarct tissue. $\mathbf{c}$ The Masson staining of myocardial tissues. The arrow shows the fibrosis. Bar $=100 \mu \mathrm{m}$ 


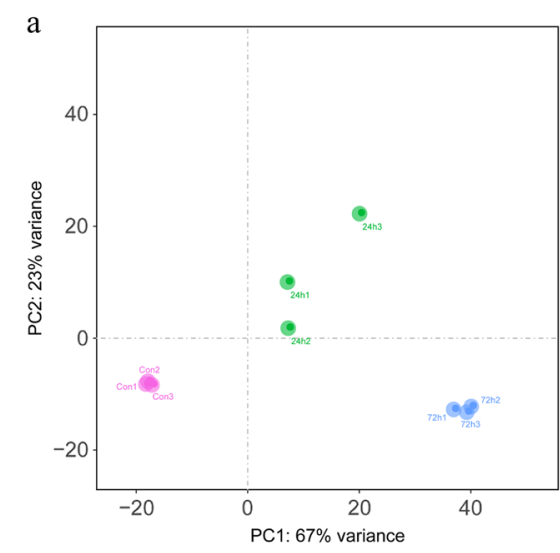

$\mathrm{c}$

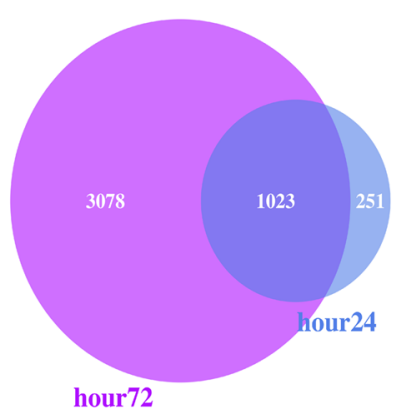

$\mathrm{b}$

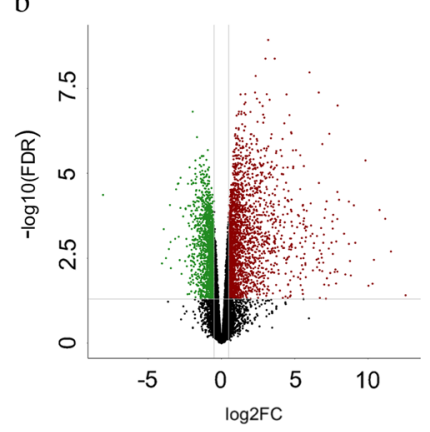

24hour

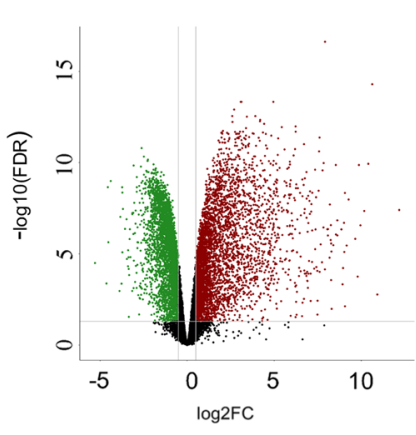

72 hour

d

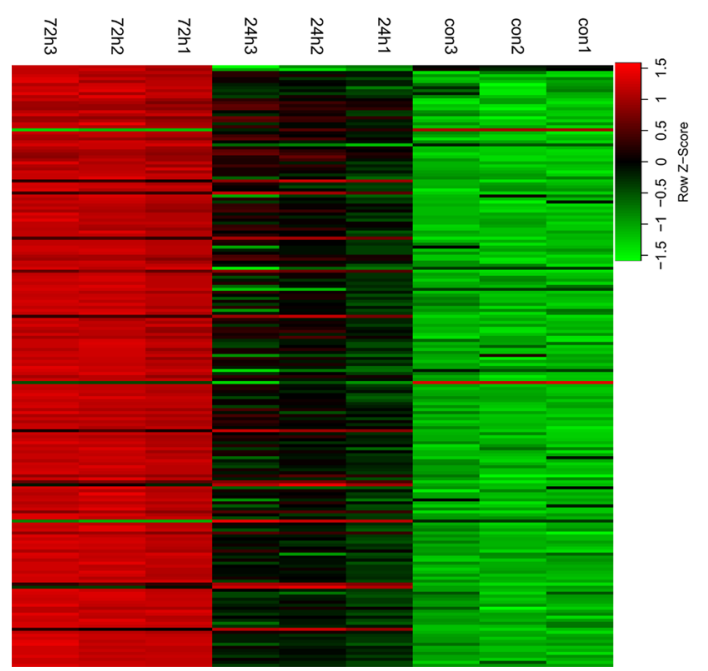

Fig. 2 Transcriptome analysis of different time points of MI. a Principal component analysis of control and Ml groups. $\mathbf{b}$ Volcano plots of the differentially expressed genes between the control group and Ml groups. $\mathbf{c}$ Venn diagram of differentially expressed genes between the control group and Ml group. $\mathbf{d}$ Heatmap of the top 200 differentially expressed genes between the control group and Ml groups

\section{Significantly increasing expression of genes involved in immunity and proliferation after MI}

We performed Gene Ontology (GO) analysis of the DEGs between control and $24 \mathrm{~h}$ groups. The results showed that more than half of the top 20 categories were associated with immunity, including inflammatory response, immune response, and chemotaxis (Fig. 3a). To further validate this result, we performed GSEA. Consistent with the GO analysis, immune-related pathways also increased significantly. These findings suggest that the immune system was deeply involved in the early stage of MI process (Fig. 3b). Then, we analysed the DEGs between control and $72 \mathrm{~h}$ groups and found that, in addition to immunity, cell cycle-related pathways and extracellular matrix remodelling-related pathways were also significantly altered (Fig. 3c, d). The above Masson staining showing partial fibrosis at $72 \mathrm{~h}$ agreed with this result.

\section{Immune cell infiltration during MI}

Our data above implicated immunity-associated pathways in the development of MI. Therefore, we focussed on the immune cell infiltration during different MI stages in the following analysis. We selected 16 different immune cell populations for the analysis, and a total of 278 genes were selected as cell-specific markers to define different immune cell signatures according to previous research (Supplementary Table 1). The heatmap showed that these cell-specific markers could distinguish these immune cell populations well (Fig. 4a). Then, we analysed the changes in different immune cell infiltration levels during MI. From the innate immune system, we found that the mRNA $z$-scores of natural killer cells (NK cells), neutrophils cells, and monocytes increased significantly after $24 \mathrm{~h}$ of MI compared with the control group (Fig. 4b, Supplemental Fig. 1a and 1c). At $72 \mathrm{~h}$, there was a more significant increase in all kinds of cells, suggesting that 


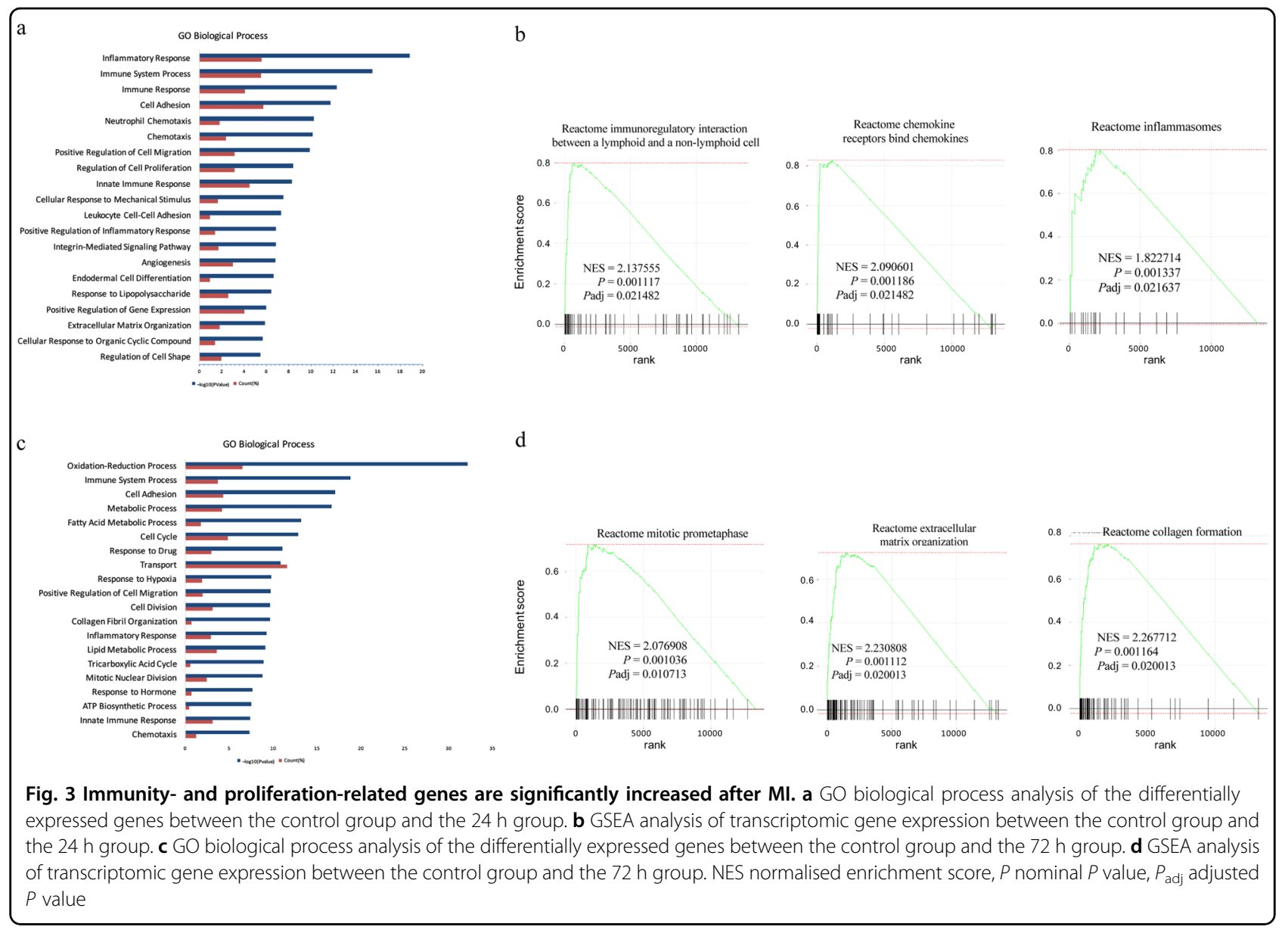

immune cells continue to accumulate in the infarct tissue during the development of MI (Fig. 4b, Supplemental Fig. 1a and 1c). We also analysed the changes in adaptive immune cells. Similarly, some kinds of cells were significantly increased in the $24 \mathrm{~h}$ group, such as naive B cells and naive $\mathrm{CD}^{+} \mathrm{T}$ cells, while in the $72 \mathrm{~h}$ group, all kinds of adaptive immune cells were significantly increased compared to the control group (Fig. 4c, d, Supplemental Fig. 1b). These results suggest that the immune cells are deeply and actively involved in the MI process.

\section{Proteome analysis}

We then analysed the tissue of the MI at different times by DIA-MS. The results showed that the expression of certain proteins changed significantly during the development of MI. Volcano plots showed that some protein levels were significantly different between the $24 \mathrm{~h}$ group and the control group, and most of them were increased in expression. In the $72 \mathrm{~h}$ group, many proteins changed significantly, and most of them were increased in expression as well (Fig. 5a). Most (85\%) of the proteins that were differentially expressed in the $24 \mathrm{~h}$ group remained significantly different in the $72 \mathrm{~h}$ group compared with control (Fig. 5b). These data show that the tissues of the three groups could be well distinguished via these proteins, suggesting a different protein expression pattern in different MI stages (Fig. 5c). We performed functional annotation of these co-variant proteins via the DAVID database (Fig. 5d). The results showed that a portion of them were closely related to immunity. Interestingly, some of these proteins were also associated with fibrinolysis. These results were consistent with the RNAseq findings above.

\section{Conjoint analysis of proteome and transcriptome}

Next, we performed a conjoint analysis of the proteome and transcriptome. Some $25 \%$ of the differentially expressed proteins also showed significant changes in the transcriptome profile between control and $24 \mathrm{~h}$ groups (Fig. 6a). Approximately $40 \%$ of the differentially expressed proteins were also differentially expressed in the transcriptome profile between control and $72 \mathrm{~h}$ groups (Fig. 6b). We then analysed the genes and proteins that changed simultaneously in both the proteome and transcriptome data, which may serve as molecular markers of MI (Fig. 6c). Nppa, an important clinical diagnostic 


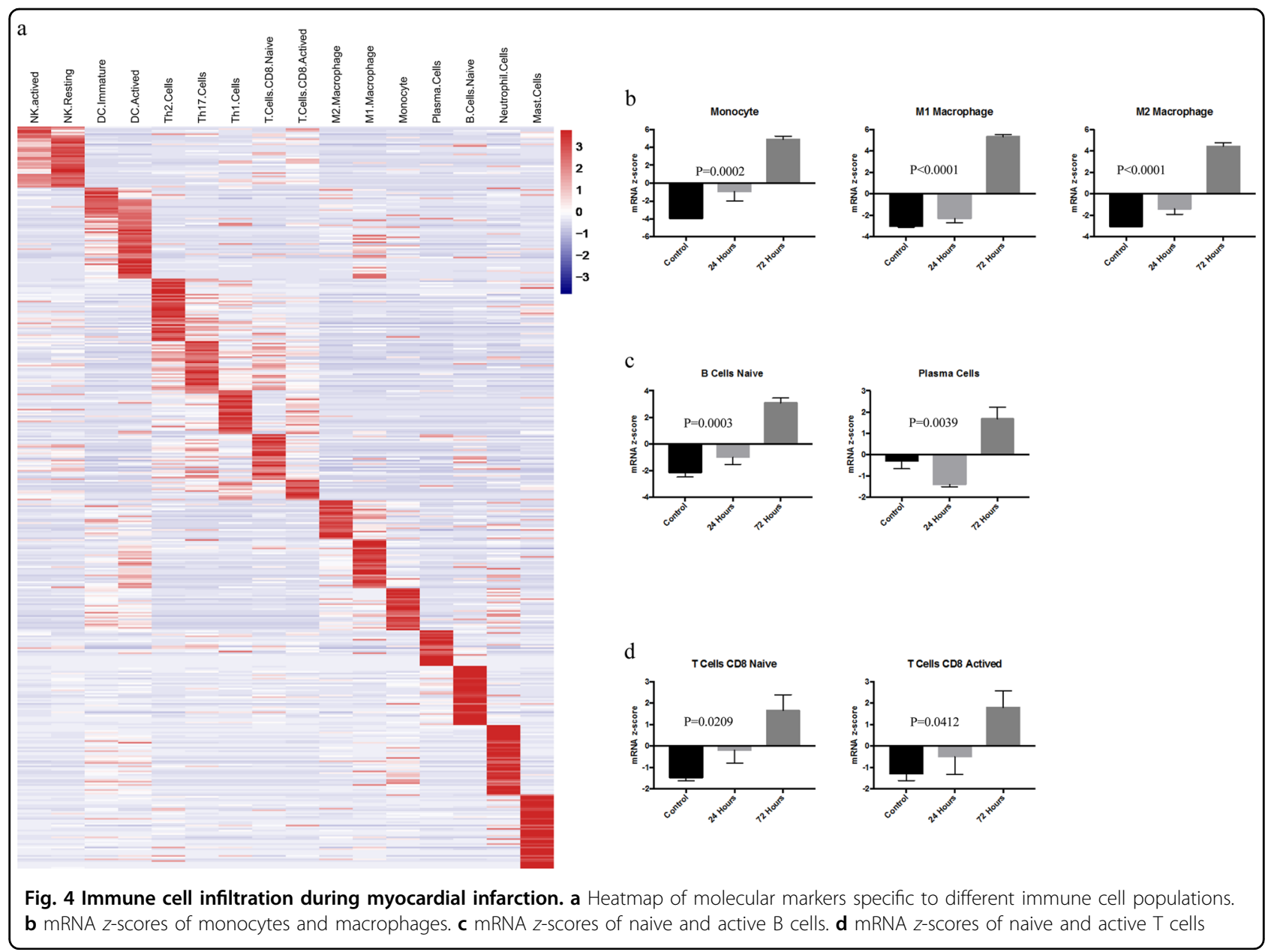

indicator of heart failure, significantly increased in the proteome and transcriptome of $\mathrm{MI}^{13}$. Serpina3n and Anxa1 also underwent significant changes at various stages during this period. Finally, we validated these results by qPCR (Fig. 6d). The results were consistent with the above analyses. Taken together, our findings suggest that these genes may be potential indicators for MI.

\section{Discussion}

In this study, we detected the gene and protein expression profile in infarct tissues of different stages through transcriptome and proteome data. We found that immune-related genes are one of the most DEG groups after MI. Not only innate immune cells but also adaptive immune cells participated in this process actively, suggesting that the infiltration of immune cells in MI tissues is an important physiopathologic process during MI. There are numerous immune cells in myocardial tissue $^{14,15}$. Though our work confirmed the involvement of immune cells in MI, the biological roles of each immune cell population and the interactions between them need to be identified by further studies. In addition to immune- related genes, genes involved in cell proliferation were also highly differentially expressed in MI tissues. The adult mouse myocardium is mainly composed of cardiomyocytes and fibroblasts. The cardiomyocytes have negligible regenerative capacity. Fibroblasts account for $60 \%$ of the total cells, and they could be stimulated to proliferate after $\mathrm{MI}^{16}$. We observed that the genes related to fibrosis were significantly up-regulated at $72 \mathrm{~h}$ of $\mathrm{MI}$ (Fig. 3d). Fibroblasts not only serve as matrix-producing reparative cells but also exhibit a wide range of functions, including inflammatory and immune response ${ }^{16,17}$. These findings, together with our work, suggest that fibroblasts may be major participants in MI.

The pathogenesis of MI is complex and multifactorial. We found that during the early stage of MI, blood agglutination-related proteins underwent significant changes, so they may be involved in the ischaemic cascade of coagulation activation. Immediate interventional treatment is recommended and routinely requires dual antiplatelet therapy, including aspirin and a P2Y12 inhibitor $^{18}$. The fibrinolysis-related pathways were also activated at the protein level, so it seems that the 


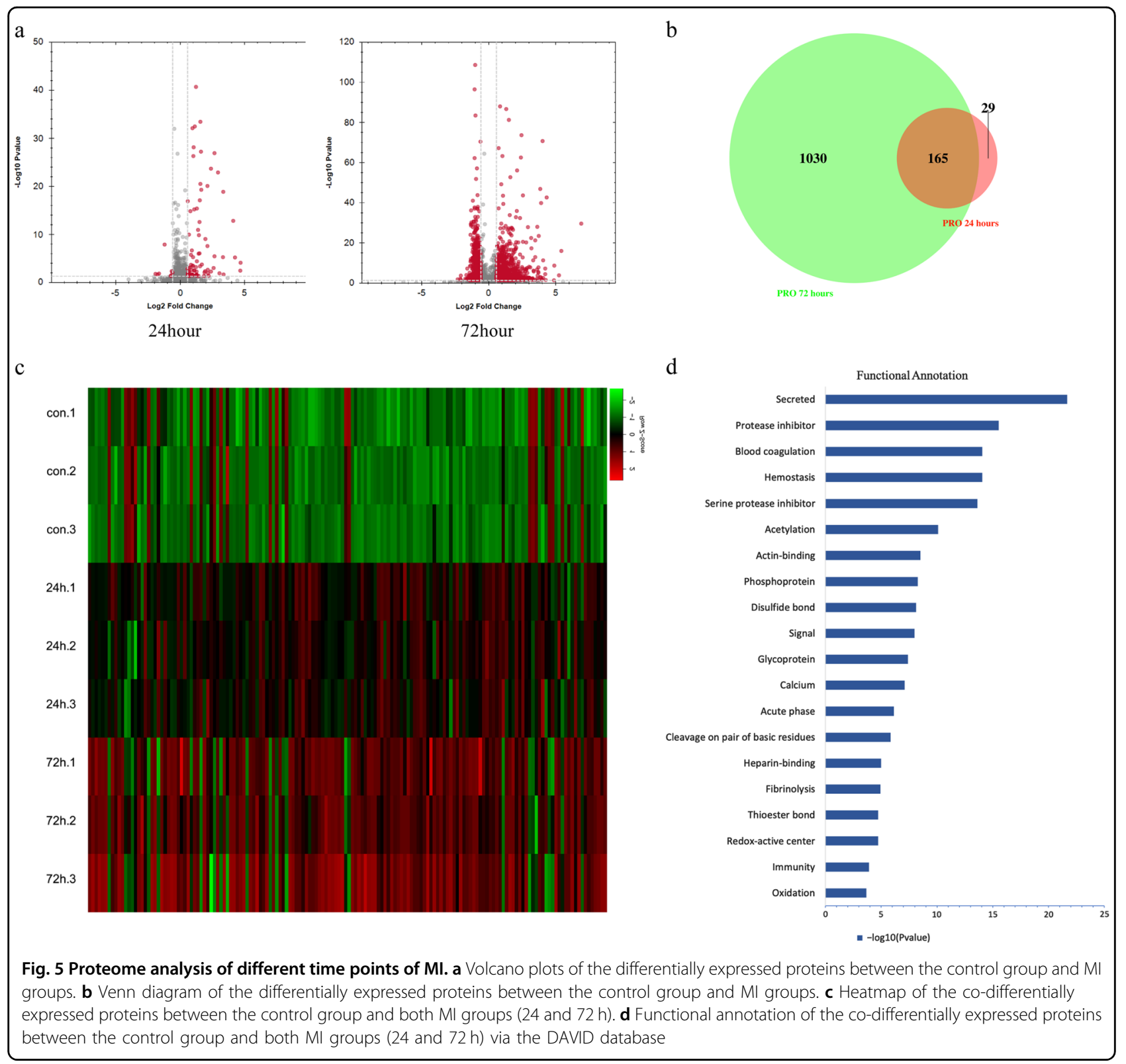

protective mechanism is initiated at an early stage. Interestingly, many protease inhibitors, mainly secreted serine protease inhibitors underwent significant changes. Serine protease inhibitors are closely involved in innate and adaptive immune responses. These results further confirm the important role of the immune system in the early stages of MI. By using DIA-MS, the accuracy and sensitivity of protein and peptide fragment detection are largely improved compared to MS. However, since proteins cannot be amplified like RNA, there is still a gap in the depth and breadth between proteome and transcriptome. Therefore, it is necessary to combine these two methods to investigate the effects of expression changes on cellular and molecular processes.
Through multi-omics analysis, we found that genes such as Serpina3n, Nppa, and Anxa1 had significant changes at both omics levels during MI. Serpina3n, a serine protease inhibitor, is released into the circulation during muscle atrophy ${ }^{19}$. Anxa1, a calcium-dependent phospholipid-binding protein, acts in immune system modulation and cell membrane organisation ${ }^{20-22}$. Nppa is a diagnostic marker for heart failure. Interestingly, all three proteins are secreted proteins and can be detected easily in plasma. Therefore, these proteins are promising biomarkers of MI for diagnosis or treatment. Further clinical investigations are needed to reveal the relationships of these proteins with MI and to screen out valuable biomarkers. 


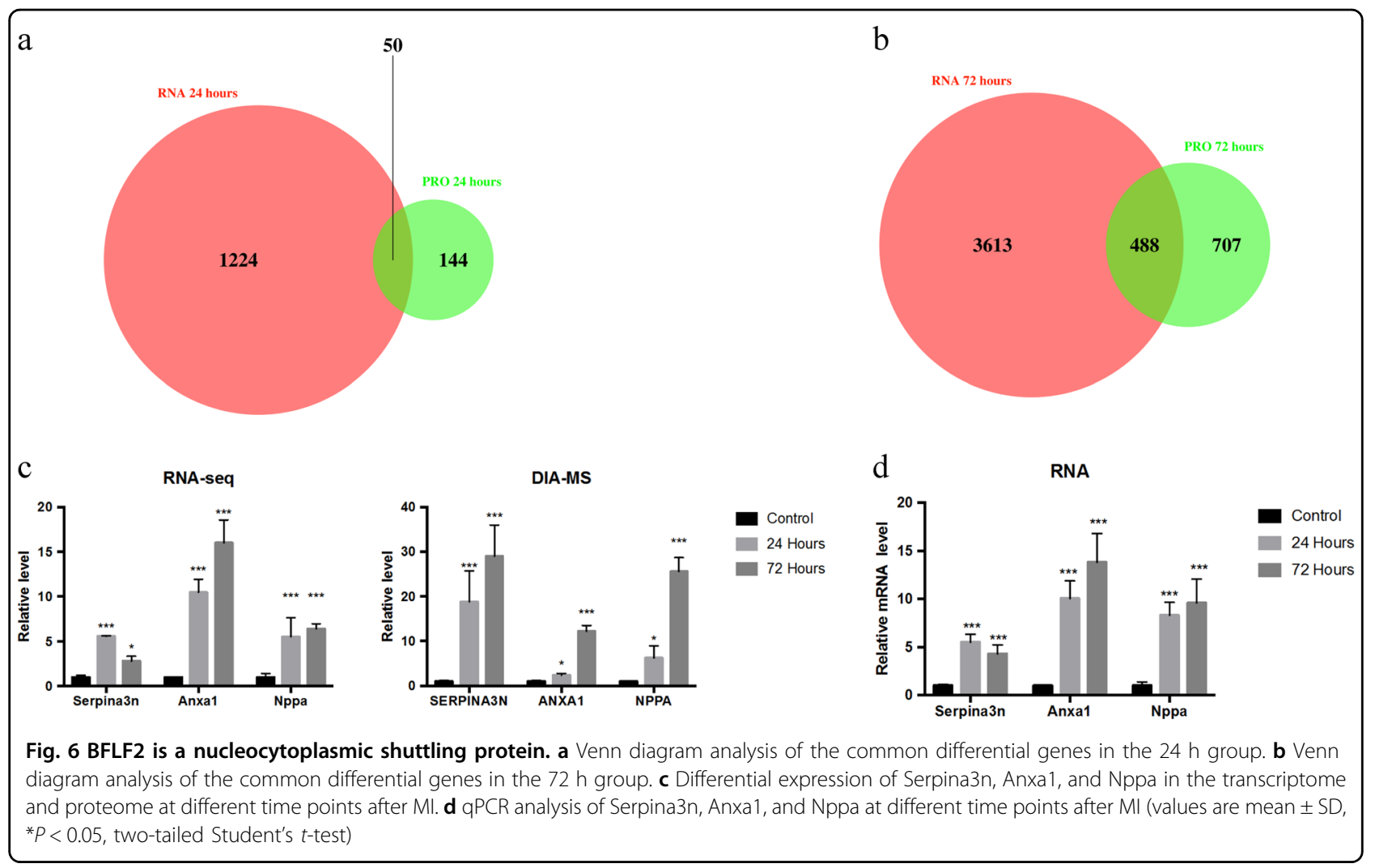

In summary, this study analysed transcriptome and proteome changes at different time points in MI. Immune-related pathways, cell cycle-related pathways, and the collagen formation pathway were significantly changed after MI. We have demonstrated that immune cells participate in the early stage of MI as well. Our findings not only reveal the molecular and cellular changes in MI but also indicate three valuable biomarkers of MI for clinical diagnosis or treatment.

\section{Materials and methods}

\section{Animals}

All mouse studies were performed in accordance with institutional guidelines for the ethical care and use of laboratory animals and were approved by the University Committee on the Care and Use of Laboratory Animals at Shanghai University of Medicine and Health Science (Shanghai, China).

Nine male C57BL/6 mice (8 weeks old) were purchased from Shanghai Slake Experimental Animal Co., Ltd. The mice were divided into three groups, the sham group, the $24 \mathrm{~h}$ MI group, and the $72 \mathrm{~h}$ MI group. They were anaesthetised by intraperitoneal injection of sodium pentobarbital $(50 \mathrm{mg} / \mathrm{kg})$. MI was performed by ligation of the proximal left anterior descending coronary artery. Then, the mice were sacrificed, and the infarcted myocardia was obtained for experiments.

\section{Quantitative reverse transcription-polymerase chain reaction (qRT-PCR)}

Total RNA was isolated using the RNAiso Reagent (TaKaRa, Japan) according to the manufacturer's instructions. Five hundred nanograms of total RNA was reverse-transcribed using the PrimeScript RT reagent kit $(\mathrm{TaKaRa})$, and resulting cDNAs were analysed by realtime qPCR with iTaq Universal SYBR Green Supermix (Bio-Rad) and SYBR Green qPCR Master Mix (Bio-Rad). Primer sequences are listed in Supplementary Table 1. The fold changes were analysed via the $2^{-\triangle \triangle \mathrm{CT}}$ method.

\section{Gene expression analysis}

Five hundred nanograms of total RNA was used for RNA-seq. Libraries were constructed (NEBNext Ultra RNA Library Prep Kit for Illumina, NEB), and size selection was performed with AMPure XP Beads (Beckman Coulter, Krefeld, Germany). Thirteen PCR cycles were used for library amplification. Amplicons were sequenced on a HiSeq 4000 (150 bp, Illumina, San Diego, USA). RNA-seq data were mapped to the GRCm38.p6 genome.

\section{Differentially expressed gene analysis}

The DEGs between control, 24-h, and 72-h groups were analysed by the edgeR R/Bioconductor package ${ }^{23}$. Genes with $\log _{2}|\mathrm{FC}| \geq 1$ and $P<0.01$ were considered to be 
significant DEGs. The DEG data are listed in Supplementary Table 1. GO analysis of DEGs was performed via The Database for Annotation, Visualization and Integrated Discovery (DAVID) (https://david.ncifcrf.gov/) ${ }^{24}$.

\section{Gene set enrichment analysis}

Fast Gene Set Enrichment Analysis (fGSEA) was performed via the fgsea $\mathrm{R} /$ Bioconductor package. The $\log _{2}(\mathrm{FC})$ value of each genes calculated by the edge $R$ package was used as the ranking metric input. We used the REACTOME pathways in the $\mathrm{C} 2$ collection in the Molecular Signatures Database (MSigDB) as the gene sets in the analysis. This sub-collection contained 674 gene sets, which were annotated pathways from the REACTOME database. The gene sets contained in the MSigDB were from a wide variety of sources and related to a variety of species, mostly human. Therefore, we downloaded the Mouse version of the MSigDB, which mapped all gene sets to mouse orthologues, from http://bioinf.wehi.edu.au/software/ MSigDB/index.html.

\section{Immune cell population analysis}

Various immune cell signatures containing genes specific to these cells can define different immune cell phenotypes (Supplementary Table 1$)^{25}$. The mRNA $z$-scores of immune cell signatures were calculated through the use of normalised RNA sequencing data via the function scale in $\mathrm{R}$ to evaluate the immune cell infiltration in myocardial tissue $^{26}$.

\section{DIA-MS}

Nine myocardium samples were lysed with lysis buffer (1\% SDS, $7 \mathrm{M}$ urea, $1 \times$ Protease Inhibitor Cocktail (Roche Ltd. Basel, Switzerland)) and centrifuged at 15,000 rpm for $15 \mathrm{~min}$ at $4{ }^{\circ} \mathrm{C}$. The supernatant was collected, and the protein concentration of the supernatant was tested by using the BCA protein assay (Beyotime Ltd. Shanghai, China). The protein samples were adjusted to $100 \mu \mathrm{l}$ with $8 \mathrm{M}$ urea. Then, $2 \mu \mathrm{l} 0.5 \mathrm{M}$ TCEP was added, and the samples were incubated at $37^{\circ} \mathrm{C}$ for $1 \mathrm{~h}$. Next, $4 \mu \mathrm{l} 1 \mathrm{M}$ iodoacetamide was added to each sample, followed by incubation for $40 \mathrm{~min}$ in the dark at room temperature. After that, five volumes of $-20^{\circ} \mathrm{C}$ pre-chilled acetone was added to precipitate the proteins overnight at $-20^{\circ} \mathrm{C}$. The precipitates were washed by $1 \mathrm{ml}$ pre-chilled $90 \%$ acetone aqueous solution twice and then re-dissolved in $100 \mu \mathrm{l}$ $100 \mathrm{mM}$ TEAB. Sequence-grade modified trypsin (Promega, WI, USA) was added at the ratio of 1:50 (enzyme: protein, weight:weight) to digest the proteins at $37^{\circ} \mathrm{C}$ overnight. The peptide mixtures were desalted by a $\mathrm{C} 18$ ZipTip, quantified by the PierceTM Quantitative Colorimetric Peptide Assay (23275) and then lyophilised by a SpeedVac.
The lyophilised peptide mixtures were re-dissolved in buffer A (20 mM ammonium formate, $\mathrm{pH}$ 10.0, adjusted with ammonium hydroxide), then fractionated by high$\mathrm{pH}$ separation using an Ultimate 3000 system (Thermo Fisher Scientific, MA, USA) connected to a reverse-phase column (XBridge C18 column), $4.6 \mathrm{~mm} \times 250 \mathrm{~mm}, 5 \mu \mathrm{m}$, (Waters Corporation, MA, USA). High-pH separation was performed with a linear gradient, starting from $5 \% \mathrm{~B}$ to $45 \% \mathrm{~B}$ in $40 \mathrm{~min}$ (B: $20 \mathrm{mM}$ ammonium formate in $80 \%$ $\mathrm{ACN}, \mathrm{pH} 10.0$, adjusted with ammonium hydroxide). The column was re-equilibrated at the initial condition for $15 \mathrm{~min}$. The column flow rate was maintained at $1 \mathrm{ml} / \mathrm{min}$, and the column temperature was maintained at $30^{\circ} \mathrm{C}$. Ten fractions were collected and each fraction was dried in a vacuum concentrator for the next step.

Then the peptides were re-dissolved in $30 \mu \mathrm{l}$ solvent $\mathrm{A}$ $(0.1 \%$ formic acid in water $)$ and analysed by on-line nanospray LC-MS/MS on an Orbitrap Fusion Lumos (Thermo Fisher Scientific, MA, USA) coupled to a Nano ACQUITY UPLC system (Waters Corporation, MA, USA). $10 \mu \mathrm{l}$ of peptides were loaded onto the trap column (Thermo Fisher Scientific) with a flow rate of $300 \mathrm{nl} / \mathrm{min}$ and subsequently separated on the analytical column (Acclaim PepMap C18, $75 \mu \mathrm{m} \times 15 \mathrm{~cm}$ ) with a nonlinearly increased gradient from $3 \% \mathrm{~B}(0.1 \%$ formic acid in $\mathrm{ACN})$ to $7 \% \mathrm{~B}$ in the first $3 \mathrm{~min}$, from $7 \% \mathrm{~B}$ to $20 \% \mathrm{~B}$ in minutes $3-83$, from $20 \%$ B to $32 \%$ B in minutes $83-107$, and from $32 \%$ B to $90 \%$ B in minutes $107-108$, followed by holding at $90 \% \mathrm{~B}$ for $12 \mathrm{~min}$. The column temperature was $40^{\circ} \mathrm{C}$. An electrospray voltage of $2.1 \mathrm{kV}$ versus the inlet of the mass spectrometer was used. The mass spectrometer was run under data-independent acquisition mode and automatically switched between MS and MS/MS mode.

\section{Acknowledgements \\ This work was supported by the National Natural Science Foundation of China (NSFC:81600303), the Chinese Postdoctoral Science Foundation \\ (2018M632169), the Special Program for Collaborative Innovation in Shanghai University of Medicine \& Health Sciences (SPCI-17-05-001), Youth Project of Shanghai Municipal Health Commission Research Funding (20184Y0175), and Shanghai Sports Bureau Research Funding (TYSKYJ201839).}

\section{Authors' contributions}

Y.L. and J.S. designed these experiments. Y.L., C.W., L.M., T.L. and F.F. performed the experiments. Y.L., C.W., Y.J. and J.S. analysed the data and compiled the figures. Y.J. and J.S. wrote the manuscript. All authors read and approved the manuscript and agreed to be accountable for all aspects of the research.

\section{Conflict of interest}

The authors declare that they have no conflict of interest.

\section{Ethics approval}

All animal experimental protocols adhered to the National Institutes of Health Guide for the Care and Use of Laboratory Animals (NIH Publications No. 8023, revised 1978) and protocols approved by the Institutional Animal Care and Use Committee of Shanghai University of Medicine \& Health Sciences (Shanghai, China). 


\section{Publisher's note}

Springer Nature remains neutral with regard to jurisdictional claims in published maps and institutional affiliations.

The online version of this article (https://doi.org/10.1038/s41420-019-0152-z) contains supplementary material, which is available to authorized users.

\section{Received: 29 January 2019 Accepted: 10 February 2019}

Published online: 04 March 2019

\section{References}

1. Reed, G. W., Rossi, J. E. \& Cannon, C. P. Acute myocardial infarction. Lancet 389 197-210 (2017).

2. Nichols, M., Townsend, N., Scarborough, P. \& Rayner, M. Cardiovascular disease in Europe 2014: epidemiological update. Eur. Heart J. 35, 2929 (2014).

3. Weintraub, W. S. et al. Value of primordial and primary prevention for cardiovascular disease: a policy statement from the American Heart Association. Circulation 124, 967-990 (2011).

4. Spath, N. B., Mills, N. L. \& Cruden, N. L. Novel cardioprotective and regenerative therapies in acute myocardial infarction: a review of recent and ongoing clinical trials. Future Cardiol. 12, 655-672 (2016).

5. Zhu, T., Yao, Q., Wang, W., Yao, H. \& Chao, J. iNOS induces vascular endothelial cell migration and apoptosis via autophagy in ischemia/reperfusion injury. Cell. Physiol. Biochem. 38, 1575-1588 (2016).

6. Ma, X. et al. Autophagy is impaired in cardiac ischemia-reperfusion injury. Autophagy 8, 1394-1396 (2012).

7. Wang, X., Guo, Z., Ding, Z. \& Mehta, J. L. Inflammation, autophagy, and apoptosis after myocardial infarction. J. Am. Heart Assoc. 7, e008024 (2018).

8. Prabhu, S. D. \& Frangogiannis, N. G. The biological basis for cardiac repair after myocardial infarction: from inflammation to fibrosis. Circ. Res. 119, 91-112 (2016).

9. Peng, Y. et al. Top-down proteomics reveals concerted reductions in myofilament and Z-disc protein phosphorylation after acute myocardial infarction. Mol. Cell. Proteomics 13, 2752-2764 (2014).

10. Yang, L. et al. Quantitative proteomics and immunohistochemistry reveal insights into cellular and molecular processes in the infarct border zone one month after myocardial infarction. J. Proteome Res. 16, 2101-2112 (2017).
11. Schmidlin, T. et al. Assessment of SRM, MRM(3), and DIA for the targeted analysis of phosphorylation dynamics in non-small cell lung cancer. Proteomics 16, 2193-2205 (2016).

12. Yuan, J. et al. Mir-21 promotes cardiac fibrosis after myocardial infarction via targeting Smad7. Cell. Physiol. Biochem. 42, 2207-2219 (2017).

13. Song, W., Wang, H. \& Wu, Q. Atrial natriuretic peptide in cardiovascular biology and disease (NPPA). Gene 569, 1-6 (2015).

14. Yan, $X$. et al. Temporal dynamics of cardiac immune cell accumulation following acute myocardial infarction. J. Mol. Cell. Cardiol. 62, 24-35 (2013).

15. Swirski, F. K. \& Nahrendorf, M. Leukocyte behavior in atherosclerosis, myocardial infarction, and heart failure. Science 339, 161-166 (2013).

16. Shinde, A. V. \& Frangogiannis, N. G. Fibroblasts in myocardial infarction: a role in inflammation and repair. J. Mol. Cell. Cardiol. 70, 74-82 (2014).

17. Frangogiannis, N. G. Regulation of the inflammatory response in cardiac repair. Circ. Res. 110, 159-173 (2012).

18. Pruller, F. et al. Impaired aspirin-mediated platelet function inhibition in resuscitated patients with acute myocardial infarction treated with therapeutic hypothermia: a prospective, observational, non-randomized single-centre study. Ann. Intensive Care 8, 28 (2018).

19. Gueugneau, M. et al. Increased Serpina3n release into circulation during glucocorticoid-mediated muscle atrophy. J. Cachexia Sarcopenia Muscle 9, 929-946 (2018)

20. Leoni, G. \& Nusrat, A. Annexin A1: shifting the balance towards resolution and repair. Biol. Chem. 397, 971-979 (2016).

21. Liu, S. et al. Annexin-1 mediates microglial activation and migration via the CK2 pathway during oxygen-glucose deprivation/reperfusion. Int. J. Mol. Sci. 17, 1770 (2016).

22. Gavins, F. N. \& Hickey, M. J. Annexin A1 and the regulation of innate and adaptive immunity. Front. Immunol. 3, 354 (2012).

23. Robinson, M. D., McCarthy, D. J. \& Smyth, G. K. edgeR: a Bioconductor package for differential expression analysis of digital gene expression data. Bioinformatics 26, 139-140 (2010).

24. da Huang, W., Sherman, B. T. \& Lempicki, R. A. Systematic and integrative analysis of large gene lists using DAVID bioinformatics resources. Nat. Protoc. 4, 44-57 (2009).

25. Chen, Z. et al. Inference of immune cell composition on the expression profiles of mouse tissue. Sci. Rep. 7, 40508 (2017).

26. Lee, E., Chuang, H. Y., Kim, J. W., Ideker, T. \& Lee, D. Inferring pathway activity toward precise disease classification. PLoS Comput. Biol. 4, e1000217 (2008). 